\title{
The syntaxins
}

\section{Felicia Yu Hsuan Teng*, Ya Wang* and Bor Luen Tang*t}

\author{
Addresses: *NCA lab and ${ }^{\dagger}$ Central Imaging and Histology Facility, Institute of Molecular and Cell Biology, 30 Medical Drive, Singapore \\ 117609, Republic of Singapore.
}

Correspondence: Bor Luen Tang. E-mail: mcbtbl@imcb.nus.edu.sg

Published: 24 October 2001

Genome Biology 200I, 2(I I):reviews30 I2.I-30I2.7

The electronic version of this article is the complete one and can be

found online at http://genomebiology.com/200I/2/II/reviews/3012

(c) BioMed Central Ltd (Print ISSN 1465-6906; Online ISSN 1465-69I4)

\begin{abstract}
Summary
The SNARE hypothesis predicts that a family of SNAP receptors are localized to and function in diverse intracellular membrane compartments where membrane fusion processes take place. Syntaxins, the prototype family of SNARE proteins, have a carboxy-terminal tail-anchor and multiple coiled-coil domains. There are 15 members of the syntaxin family in the human genome and 7 syntaxin-like genes in the yeast Saccharomyces cerevisiae. In conjunction with other SNAREs and with the cytoplasmic NSF and SNAP proteins, syntaxins mediate vesicle fusion in diverse vesicular transport processes along the exocytic and the endocytic pathway. They are crucial components that both drive and provide specificity to the myriad vesicular fusion processes that characterize the eukaryotic cell.
\end{abstract}

Traffic between intracellular membrane compartments is largely mediated by vesicular transport. High degrees of specificity and complexity are exerted in the regulation of vesicle budding, docking and fusion. In a breakthrough, Rothman and colleagues showed in a cell-free assay that the docking and fusion of transport vesicles require the concerted action of two cytosolic proteins: $N$-ethylmaleimidesensitive factor (NSF), an ATPase whose activity regulates the formation and dissociation of the complexes needed for membrane fusion, and an additional factor needed to attach NSF to Golgi membranes, the soluble NSF-attachment protein, SNAP (reviewed in [1]).

Factors that determine the specificity of the docking and fusion of vesicles to the correct target membranes were subsequently identified from their incorporation, again in a cell-free assay, into a $20 \mathrm{~S}$ assembly complex with recombinant NSF and $\alpha$-SNAP [2]. These SNAP receptors, or SNAREs, turned out to be previously cloned components of the synaptic membrane with unknown function. Remarkably, these SNAREs share structural homology with several yeast genes whose products are associated with vesicular transport. These findings pointed towards a conserved mechanism in the diverse transport processes amongst eukaryotic cells. To account for the specificity of each step, the SNARE hypothesis
[3] postulated the existence of a family of molecules, with each molecule functioning in one of the various membranefusion processes in the cell. One of the molecules identified as a SNARE from bovine brain was a syntaxin.

\section{Gene organization and evolutionary history}

Syntaxin was first described as two $35 \mathrm{kDa}$ proteins (now known as syntaxin $1 \mathrm{~A}$ and $1 \mathrm{~B}$ ), 84\% identical to each other in amino-acid sequence, that interact with the synaptic-vesicle protein synaptotagmin [4]. It soon became clear that there are also non-neuronal homologs of syntaxin. The first syntaxin localized to the early secretory pathway, syntaxin 5, was cloned along with the cell-surface syntaxins 2, 3 and 4 [5]. More recently, syntaxins localized to the endosomes have also been identified [6]. A summary of the genetic, cellular and functional information of known mammalian and yeast syntaxins and their phylogenetic relationships is shown in Tables 1 and 2 and Figure 1.

The syntaxin family consists of 15 genes in mammals and 7 in yeast, and there are syntaxin-like sequences in all eukaryotes examined to date. The mammalian syntaxin genes are on different chromosomes (summarized in Table 1). Syntaxins 3 and 5 are both found on chromosome 11 but at different 
Table I

\begin{tabular}{|c|c|c|c|c|}
\hline Syntaxins & Chromosomal localization* & Cellular localization & Tissue distribution & Known function \\
\hline $\begin{array}{c}\text { Syntaxin IA } \\
\text { (A and C) }\end{array}$ & $7 q 11.23$ & $\begin{array}{l}\text { Presynaptic plasma } \\
\text { membrane }\end{array}$ & $\begin{array}{l}\text { Neuronal and } \\
\text { secretory cells }\end{array}$ & $\begin{array}{l}\text { Neuronal exocytosis; } \\
\text { regulated secretion }\end{array}$ \\
\hline Syntaxin IB & $|6 p| \mid .2$ & $\begin{array}{l}\text { Presynaptic plasma } \\
\text { membrane }\end{array}$ & $\begin{array}{l}\text { Neuronal and } \\
\text { secretory cells }\end{array}$ & $\begin{array}{l}\text { Neuronal exocytosis; } \\
\text { regulated secretion }\end{array}$ \\
\hline $\begin{array}{l}\text { Syntaxin } 2 \\
\text { (A, B, C and D) }\end{array}$ & 7 & Plasma membrane & Ubiquitous & $\begin{array}{l}\text { Exocytosis; morphoregulator } \\
\text { during development }\end{array}$ \\
\hline $\begin{array}{l}\text { Syntaxin } 3 \\
\text { (A, B, C and D) }\end{array}$ & II cen-I IqI 2.3 & Plasma membrane & Ubiquitous & Exocytosis \\
\hline Syntaxin 4 & $16 p|3.13-16 p| 2.3$ & Plasma membrane & Ubiquitous & Glut4 translocation \\
\hline $\begin{array}{l}\text { Syntaxin } 5 \\
\text { (long and short form) }\end{array}$ & IIcen-IIqI2.I & ER-Golgi boundary & Ubiquitous & ER-Golgi transport \\
\hline Syntaxin 6 & 1 & TGN & Ubiquitous & $\begin{array}{c}\text { TGN-endosome transport; } \\
\text { endosome-TGN transport; } \\
\text { fusion of immature secretory granules }\end{array}$ \\
\hline Syntaxin 7 & 6 & Endosome & Ubiquitous & $\begin{array}{l}\text { Late endosome fusion; } \\
\text { late endosome-lysosome fusion }\end{array}$ \\
\hline Syntaxin 8 & $|7 p| 2$ & Endosome & Ubiquitous & Late endosome fusion \\
\hline Syntaxin 10 & $19 p \mid 3.2$ & TGN & Ubiquitous & $?$ \\
\hline Syntaxin II & $6 q 23.1-6 q 25.3$ & TGN/late endosome & Ubiquitous & $?$ \\
\hline Syntaxin $12 / 13$ & 1 & Endosome & Ubiquitous & $\begin{array}{l}\text { Recycling of surface protein; } \\
\text { early endosome fusion }\end{array}$ \\
\hline $\begin{array}{l}\text { Syntaxin } 16 \\
(A, B \text { and } C)\end{array}$ & $20 p|| .23-20 p|| .2 \mid$ & Golgi/TGN & Ubiquitous & Early endosome-TGN transport \\
\hline Syntaxin 17 & $?$ & Smooth ER & Steroidogenic tissues & Trafficking to smooth ER \\
\hline Syntaxin 18 & 4 & ER & $?$ & $\begin{array}{l}\text { ER-Golgi transport; } \\
\text { ER homotypic fusion }\end{array}$ \\
\hline
\end{tabular}

Alternatively spliced isoforms, if known, are shown in brackets after each syntaxin. *The chromosomal localizations of mammalian syntaxins that are indicated are those of human, and are based on the information at the LocusLink site [15] of the National Center for Biotechnology Information (NCBI).

loci. Additional diversity within the syntaxin family is generated by alternative splicing; alternatively spliced isoforms have been identified for syntaxins 1A, 2, 3, 5 and 16. The domains that can be removed or included by alternative splicing commonly include the membrane-proximal domain of syntaxin, the region required for SNARE complex assembly and/or the carboxy-terminal hydrophobic membrane anchor. The splice isoforms are differentially expressed during development and in different tissues in adult life, and may thus have substantially different functional roles in the regulation of membrane traffic.

\section{Characteristic structural features}

All mammalian syntaxins, with the exception of syntaxin 11, are transmembrane proteins anchored by their carboxy-terminal tails with a type II orientation (that is, with the amino terminus and the bulk of the polypeptide facing the cytoplasm). The domain structure of syntaxin $1 \mathrm{~A}$, the first to be identified, is shown schematically in Figure 2b. Other than the transmembrane domain, there are several hydrophobic regions (Figure 2a) with the potential to form coiled-coil $\alpha$-helical structures. The approximately 6o-residue-long membrane-proximal coiled-coil domain is the SNARE domain, which is characteristic of and conserved in all syntaxins [7].

The SNARE domain of a syntaxin mediates its interactions with the SNARE domains of other target-membrane ( $\mathrm{t}$ ) SNARE proteins from the syntaxin or SNAP-25 families, to form t-SNARE complexes at target membranes. The t-SNARE complexes, in turn, interact with SNARE domains of the vesicle (v) SNAREs (vesicle-associated membrane proteins, VAMPs) found on specific vesicle membranes, to form the core fusion complex. Perhaps the best-studied membrane-fusion complex is that mediating synaptic-vesicle fusion [8-10]. An extremely stable ternary complex, a $12 \mathrm{~nm}$ long twisted bundle of four helices aligned in parallel, is 
Table 2

\begin{tabular}{lccl}
$l$ & \multicolumn{3}{l}{ Genetic, cellular and functional information about yeast syntaxins } \\
\hline Syntaxins & Cellular localization & Null phenotype & Known function \\
\hline Ssolp (YPL232W) & Plasma membrane & Viable & Transport to cell surface \\
Sso2p (YMR 183C) & Plasma membrane & Vable & Transport to cell surface \\
Ufelp (YOR075W) & ER & Lethal & ER homotypic fusion \\
Sed5p (YLR026C) & Golgi & Lethal & ER-Golgi transport; retrograde transport to Golgi \\
Tlg2p (YOL018C) & Golgi & Viable & Endosome biogenesis; cytoplasm to vacuole transport; endosomal recycling \\
PepI2p (YOR036W) & Golgi/vacuole/endosome & Viable & Vacuolar targeting \\
Vam3p (YORI06W) & Vacuole & Viable & Vacuolar targeting; phagosome fusion to vacuole
\end{tabular}

formed by syntaxin 1A, SNAP-25 and VAMP-2, which contribute one, two and one $\alpha$ helices, respectively (Figure 2c) [11]. Most other heterotypic core fusion complexes are likely to have a similar parallel four-helical bundle structure, but in some cases the two $\alpha$-helical SNARE domains provided by SNAP-25 may be replaced by the SNARE domains of two members of the syntaxin family.

The amino terminus of some syntaxins, such as syntaxin 1, contains another characteristic domain, which is thought to vary between syntaxin isoforms depending on the specific vesicle-traffic steps involved. The amino-terminal domain of syntaxin 1 (Figure 2b) is a bundle of three $\alpha$ helices with a left-handed twist [12]. This conserved autonomously folding amino-terminal structure may serve as an auto-inhibitory regulatory domain. By folding back onto the membraneproximal SNARE domain, the molecule adopts a 'closed' configuration that prevents the formation of the core fusion complex. The chaperone protein n-Sec1/Munc18 binds to this closed conformation of syntaxin. Dissociation or conformational changes in n-Sec1/Munc-18 induced by the Rab small GTPases may open up the structure to facilitate SNARE-complex formation.

\section{SNARE complex assembly and disassembly}

SNAREs, together with SNAP and NSF, form a 20 S complex intermediate that is essential for the docking and fusion of vesicles with a target membrane. As mentioned, in the case of synaptic-vesicle exocytosis, syntaxin 1 and its cognate SNARE partners form a ternary complex consisting of a coiled-coil bundle of four $\alpha$ helices, before NSF/ $\alpha$-SNAP joins to form the $20 S$ complex. These four helices are parallel with the transmembrane domains of VAMP and syntaxin, at the same end of the bundle. The formation of the $20 S$ complex thus creates a bridge between the vesicle and the target membrane. Evidence suggests that, in the initial stage of vesicle docking, the SNARE complex assumes a partial and reversible assembly known as the 'trans-conformation'. In this case, the syntaxin coil is likely to be less tightly associated than the v-SNARE and SNAP25 coils, and its full association is postulated to be held back by a calcium sensor, until the arrival of $\mathrm{Ca}^{2+}$ signal. The $\mathrm{Ca}^{2+}$ trigger results in the full association of the syntaxin coil, converting the transcomplex into a tight 'cis-complex'. In a 'zipper' model of SNARE-mediated fusion, it is postulated that the transcomplex might zipper up from the amino terminus towards the carboxy-terminal transmembrane end, bringing the two membranes together and thereby causing the final membrane fusion.

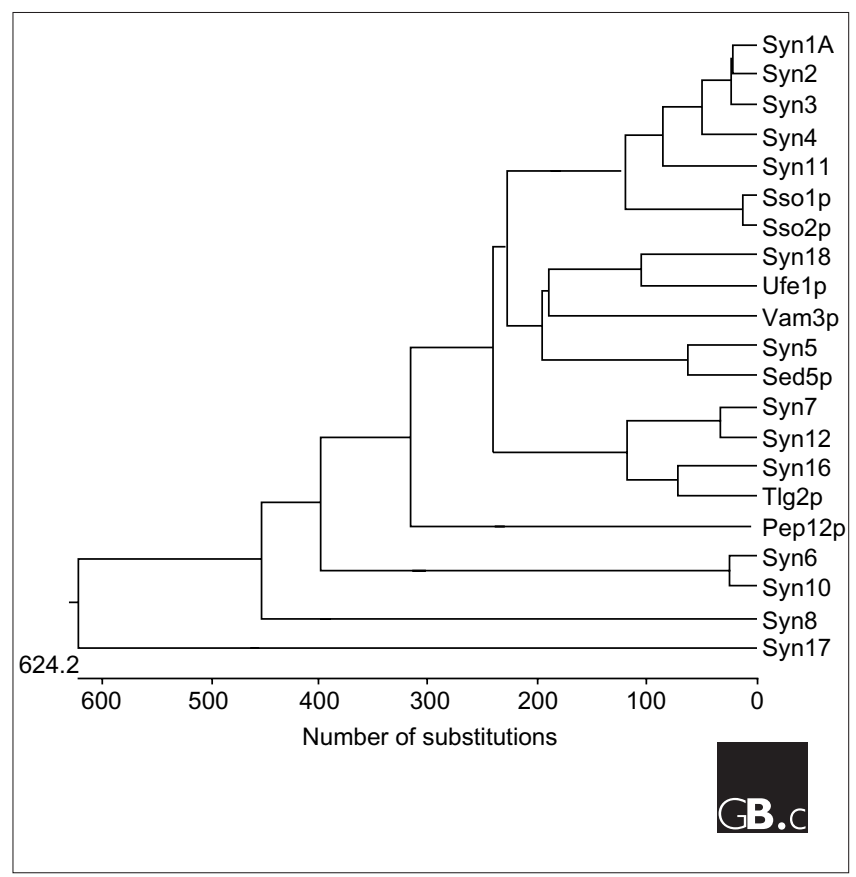

Figure I

A nearest-neighbor dendrogram of the $7 \mathrm{~S}$. cerevisiae and 15 mammalian syntaxins, generated with the DNASTAR program. See Table 2 for yeast gene names; syn, syntaxin. There are also several syntaxin-like genes in the Drosophila and $C$. elegans genome; see Bock et al. [6] for a more extensive phylogenetic analysis that includes these sequences. 
(a)

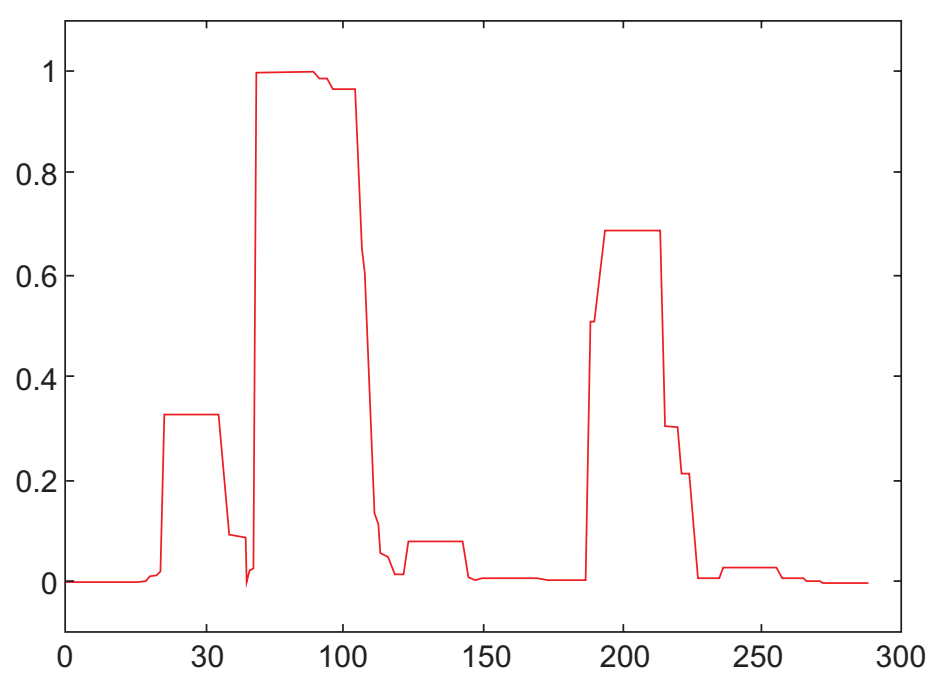

(b)

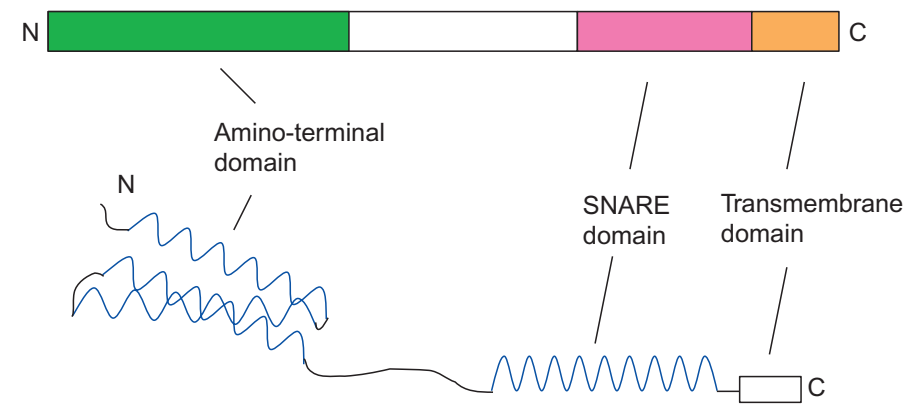

(c)

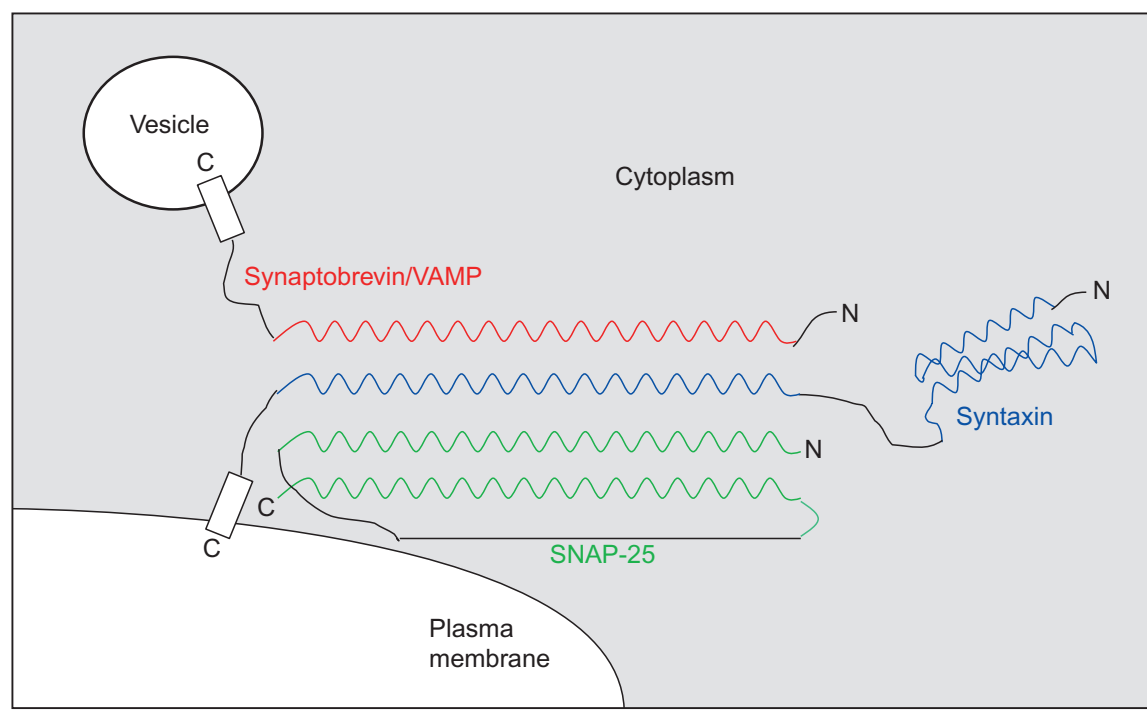

Figure 2

The structure of syntaxin and a syntaxin-containing SNARE complex. (a) A graphical output of the analysis with the COILS program [16] of syntaxin IA, for potential coiled-coil-forming regions. A window size of 21 residues was used. (b) Schematic representation of the structure of syntaxin IA, illustrating both the linear domain arrangement (upper diagram) and the coiled-coil domains (blue) with spatial relevance to one another (lower diagram). N, amino terminus; C, carboxyl terminus. (c) Schematic representation of the four-helical bundle structure of the core fusion complex formed by syntaxin IA (blue), synaptobrevin/VAMP (red) and SNAP-25 (green) at the presynaptic plasma membrane. 
The cis-complex formed after vesicle docking and membrane fusion is then dissociated by NSF and its co-factor $\alpha$-SNAP. The hexameric NSF has two ATP-binding sites (D1 and D2) per subunit. The binding of $\alpha$-SNAP to NSF stimulates nucleotide hydrolysis at the D1 sites. NSF undergoes a conformational change upon ATP hydrolysis, which provides a mechanical force for disassembling the SNARE complex. The disassembly of the complex by NSF frees the SNAREs for recycling and the formation of a new trans-complex for the next round of vesicle docking and fusion.

\section{Localization and function}

A summary of the cellular localization of mammalian and yeast syntaxins can be found in Tables 1 and 2 and Figure 3 . Cellular localizations for all mammalian syntaxins have been confirmed using antibodies against endogenous proteins. It is worth noting that, although transgenic syntaxins epitopetagged at the amino terminus generally retain the localization of the endogenous protein, overflowing of the proteins to neighboring compartments as a result of overexpression can complicate interpretation of localization experiments. On the other hand, morphological changes to particular cellular compartments - due to transport defects or otherwise as a result of overexpression of a particular syntaxin or its mutant can also be informative with regard to its localization and site of function.

The transmembrane tail anchor found in all syntaxins except syntaxin 11 is essential for their membrane localization (exogenously expressed cytoplasmic domains appear to be cytosolic), but in most cases it is not sufficient for specific targeting to particular membranes. Compartmental targeting signals reside in the cytoplasmic domains, and these are not well defined. An examination of the amino-acid sequences of syntaxins that are found in the trans-Golgi network (TGN) and post-Golgi compartments has revealed putative targeting signals with dileucine motifs, but these have not, in most cases, been shown to be functional by mutational analysis.

The SNARE hypothesis predicts that, as a t-SNARE, the cellular function of a particular syntaxin would logically be determined and restricted by its localization. Thus, it is not surprising to find that syntaxin 18 , which is localized to the endoplasmic reticulum (ER), and the cis-Golgi-localized syntaxin 5 have roles in ER-to-Golgi transport. In fact, the lack of a syntaxin within the Golgi stack itself has been suggested as indirect evidence for the maturation model of intra-Golgi transport (in which Golgi compartments move from ER to TGN as they mature, as opposed to the vesicular transport model, in which vesicles shunt between 'static' compartments). On the other hand, one explanation for the apparent multiple functions of the predominantly TGNlocalized syntaxin 6 is that it is perhaps not a true syntaxin, even though it has been categorized as one, as its SNARE domain resembles that of the SNAP23/25/29 family members more than the syntaxins [6]. The exact roles of the endosomal syntaxins (syntaxin 7, 8, 11 and 12/13) are not particularly well defined. One reason for this is the lack of biochemical assays to precisely dissect the complicated steps in endosomal transport. The surface syntaxins have been implicated in various processes involving the delivery of TGN cargo to the cell surface. Syntaxin 4, for example, is essential for translocation of the glucose transporter molecule Glut4 to the plasma membrane of insulin-responsive cells.

Syntaxins have been shown to interact with a range of other proteins as well as their SNARE partners. These can be broadly classified as either components of the vesicular transport machinery or proteins with no predicted function in vesicular transport. The former include vesicle coat proteins, Rab GTPases and tethering factors. It would not be surprising or exceptionally interesting that syntaxins may interact with coat proteins, which are, after all, cargo proteins in their own right. There is evidence, however, that some v-SNAREs are initiators of coat-protein assembly and vesicle budding. This makes sense as it would ensure that vesicles are 'functional', equipped with downstream docking and fusion components. Rab proteins and their effectors are regulators of the vesicle docking and fusion processes. Multiprotein complexes such as the homotypic fusion and vacuole protein sorting (HOPS) complex interact functionally with Rabs and SNAREs to regulate docking [13]. In perhaps most instances, vesicle docking is preceded by a process known as tethering, whereby molecules known as tethering factors bring vesicles close to the target membrane, to enhance productive docking and fusion. The direct interaction between a syntaxin, syntaxin 13, and the tethering factor early endosome antigen 1 (EEA1) has been elegantly demonstrated in the case of Rab5-regulated early endosomal homotypic fusion (fusion of similar vesicles) [14].

Syntaxins may also interact with other proteins. Syntaxin $1 \mathrm{~A}$ and syntaxin 3 have been known to interact with sodium channels in epithelial cells, thereby regulating the intrinsic properties and cell-surface expression of the channels. An antibody against syntaxin $1 \mathrm{~A}$ immunoprecipitates solubilized N-type calcium channels, suggesting a role in docking synaptic vesicles near calcium channels in presynaptic active zones. The interaction between syntaxin $1 \mathrm{~A}$ and voltage-sensitive calcium channels in an 'excitosome' complex at the presynaptic plasma membrane may enable a rapid secretory response to a membrane-depolarizing signal.

\section{Frontiers}

With the solution of the structures of SNARE complexes and advanced biochemical and biophysical analysis, we now have a fair idea of how syntaxins interact with their SNARE partners and how these interactions are regulated. With the completion of the sequencing of the human genome, all human syntaxins 


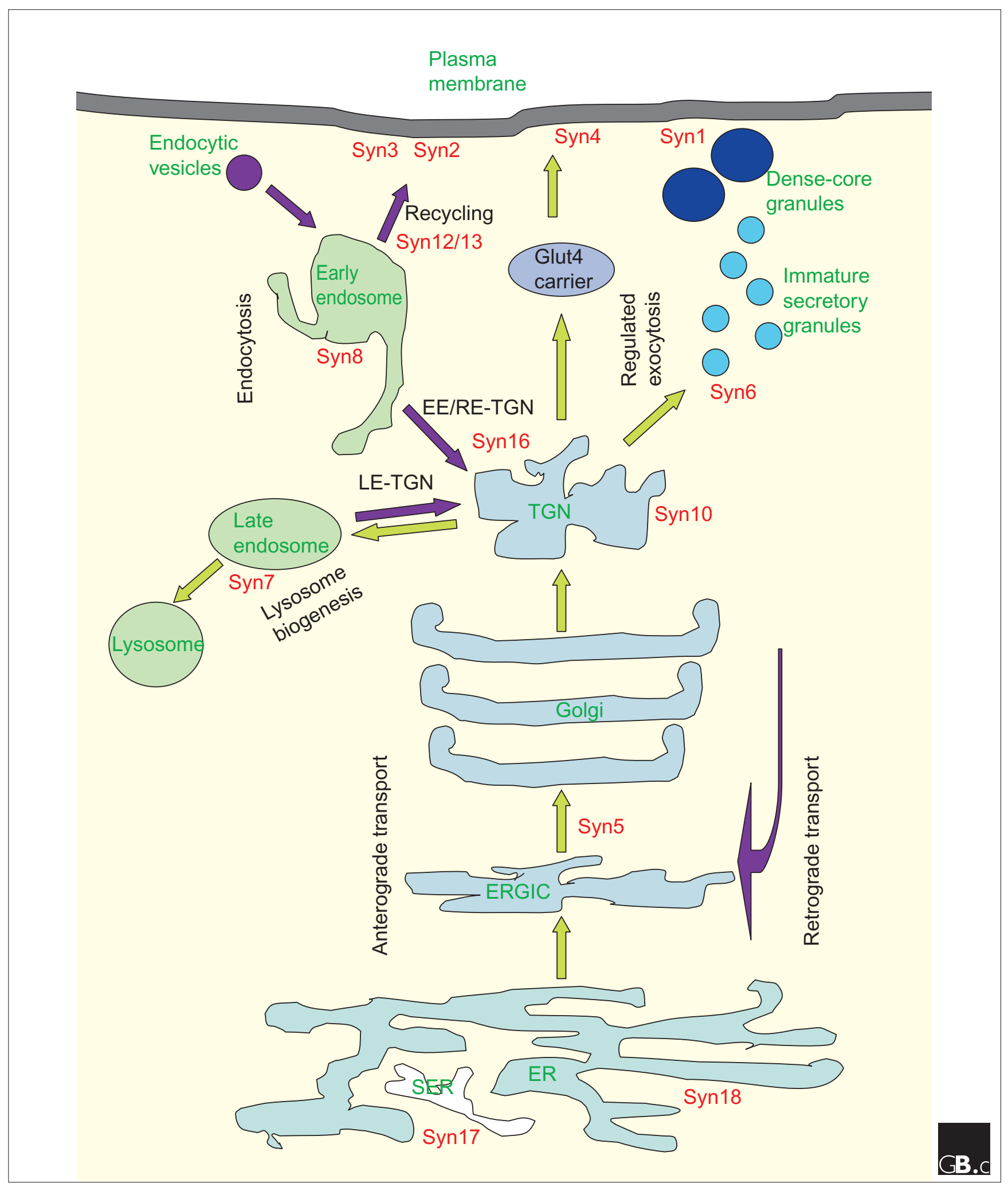

\section{Figure 3}

Subcellular localization of syntaxins (red) in a mammalian cell relative to the various membrane-bound compartments, anterograde and endocytotic/retrograde flow of traffic (green and purple arrows, respectively) and known membranetransport steps (black). EE, early endosome; ERGIC, ER-Golgi intermediate compartment; Glut4, a glucose transporter molecule; LE, late endosome; RE, recycling endosome, SER, smooth ER; syn, syntaxin; TGN, trans-Golgi network. 
have now been identified. Many of the cellular and physiological functions of syntaxins remain to be learned, however.

Firstly, the exact components of the SNARE complexes, or for that matter the exact involvement of any particular syntaxin, remains unclear in several important membrane transport steps. It is not yet known, for example, whether syntaxin 18 has a function in homotypic fusion in the ER, like the yeast homolog Ufe1p. The mammalian syntaxin 5 appears to be present both on vesicles en route to the Golgi and on the target membrane itself, and can be found in distinct SNARE complexes. It is not known if the SNARE complexes that include syntaxins 5 and 18 function in sequence or in parallel in ER-Golgi transport. Likewise, syntaxin 6 appears to serve multiple functions in transport processes in and out of the TGN that are not yet clearly defined, as well as post-TGN processes. As already mentioned, much remains to be learned about the mechanistic roles of the endosomal syntaxins. Another important line of investigation is to confirm the participation of the cell-surface syntaxins (especially syntaxin 3) in specialized processes such as neurite outgrowth and myelin sheath formation.

Secondly, although it is clear that syntaxins may have physiologically important direct or indirect physical interactions with other components of the vesicular transport system that do not belong to the core machinery of membrane fusion, much of this knowledge is fragmented. The interactions of syntaxins with Rab proteins and tethering proteins have been investigated and demonstrated only in isolated cases. Extension of this type of knowledge to other syntaxins is absolutely essential for our further understanding of the regulation of syntaxin function. Also, reports of interactions between syntaxins and other molecules that do not appear to serve general roles in transport are confined to the ion channels that interact with syntaxin 1 and 3 . Further efforts in looking for interactions of syntaxins with non-SNARE and non-transport components are warranted.

Finally, the functions of most of the syntaxins with respect to organism growth, physiology and development are absolutely unknown. It would be fair to speculate that syntaxins in the early secretory pathway of mammalian cells would be so vital that any ablation of their genes would result in death of cells, let alone of the organism. But the fact that all post-Golgi syntaxin-like molecules in yeast are not essential for growth points to the possibility that some of the mammalian postGolgi syntaxins may have physiological functions that are amenable to genetic analysis by mouse 'knockout' and 'knockin' genetic approaches. If so, it may then be possible to investigate whether these syntaxins have a role in embryonic development, post-natal growth, or the organization of particular tissues or structures. Extracting mechanistic information from the knockout phenotypes may be difficult, though. There is clearly much more to learn about the physiological functions of these important proteins.

\section{References}

I. Rothman JE: Mechanisms of intracellular protein transport. Nature 1994, 372:55-63.

A review covering most of the early mechanistic findings on vesicular transport.

2. Sollner $T$, Whiteheart SW, Brunner M, Erdjument-Bromage $H$, Geromanos S, Tempst P, Rothman JE: SNAP receptors implicated in vesicle targeting and fusion. Nature 1993, 362:3 I8-324. A landmark paper describing the purification and identification of SNAP receptors, or SNAREs.

3. Rothman JE, Warren G: Implications of the SNARE hypothesis for intracellular membrane topology and dynamics. Curr Biol 1994, 4:220-233.

An elaborate account of the SNARE hypothesis, in which the authors propose that multiple SNAREs at various membranes provide the specificity for vesicular transport.

4. Bennett MK, Calakos N, Scheller RH: Syntaxin: a synaptic protein implicated in docking of synaptic vesicles at presynaptic active zones. Science 1992, 257:255-259.

The first cloning of a syntaxin.

5. Bennett MK, Garcia-Arraras JE, Elferink LA, Peterson K, Fleming AM, Hazuka $C D$, Scheller RH: The syntaxin family of vesicular transport receptors. Cell 1993, 74:863-873.

Cloning of the first non-neuronal syntaxins.

6. Bock JB, Matern HT, Peden AA, Scheller RH: A genomic perspective on membrane compartment organization. Nature 200I, 409:839-84I.

A detailed and thorough analysis of transport-related proteins in the sequenced genomes. An excellent reference source for syntaxins.

7. Weimbs T, Low SH, Chapin SJ, Mostov KE, Bucher P, Hofmann K: A conserved domain is present in different families of vesicular fusion proteins: a new superfamily. Proc Natl Acad Sci USA 1997, 94:3046-305I.

A classic bioinformatics paper that provided the first idea of how syntaxins and VAMPs are related.

8. Chen YA, Scheller RH: SNARE-mediated membrane fusion. Nat Rev Mol Cell Biol 200I, 2:98-106.

The most up-to-date review on the mechanisms of SNARE-mediated membrane fusion.

9. Jahn R, Sudhof TC: Membrane fusion and exocytosis. Annu Rev Biochem 1999, 68:863-91I.

A detailed review of the players and mechanisms involved in exocytosis.

10. Lin RC, Scheller RH: Mechanisms of synaptic vesicle exocytosis. Annu Rev Cell Dev Biol 2000, 16:19-49

A detailed review of the players and mechanisms of synaptic-vesicle exocytosis.

II. Sutton RB, Fasshauer D, Jahn R, Brunger AT: Crystal structure of a SNARE complex involved in synaptic vesicle exocytosis at 2.4 Å resolution. Nature 1998, 395:347-353.

The first description of the crystal structure of a SNARE complex.

12. Lerman JC, Robblee J, Fairman R, Hughson FM: Structural analysis of the neuronal SNARE protein syntaxin-IA. Biochemistry 2000, 39:8470-8479.

Structural analysis of the amino-terminal domain of syntaxin IA at I.9 resolution.

13. Eitzen G, Will E, Gallwitz D, Haas A, Wickner W: Sequential action of two GTPases to promote vacuole docking and fusion. EMBO J 2000, 19:67।3-6720.

Vacuole homotypic fusion in yeast is perhaps the system in which membrane fusion mechanisms have been most studied. This paper, one of an excellent series from William Wickner's laboratory, describes how Rab GTPases regulate the process.

14. McBride HM, Rybin V, Murphy C, Giner A, Teasdale R, Zerial M: Oligomeric complexes link Rab5 effectors with NSF and drive membrane fusion via interactions between EEAI and syntaxin 13. Cell 1999, 98:377-386.

An important paper showing a direct link between a Rab protein, a tethering protein and a SNARE protein.

15. LocusLink [http://www.ncbi.nlm.nih.gov/LocusLink/] A National Center of Biotechnology Information (NCBI) program that provides a single interface for querying curated sequences and descriptive information about genetic loci.

16. COILS - prediction of coiled coil regions in proteins [http://www.ch.embnet.org/software/COILS_form.html] A program for predicting coiled-coil-forming regions, available at the EMBnet website. 